Method Leptin and adiponectin levels were measured in 388 non-diabetic officers from the Buffalo Cardio-Metabolic Occupational Police Stress study, following a 12-hour fast. HRV was performed according to methods published by the Task Force of the European Society of Cardiology and the North American Society of Pacing Electrophysiology for measurement and analysis of HRV. Mean values of high (HF) and low frequency (LF) HRV were compared across tertiles of leptin and adiponectin using ANOVA and ANCOVA; trends were assessed using linear regression models.

Results Leptin, but not adiponectin, was significantly and inversely associated with HF and LF HRV. BMI and percent body fat (also waist circumference and abdominal height) significantly modified the association between leptin and LF (but not HF) HRV. Among officers with BMI $<25 \mathrm{~kg} / \mathrm{m}^{2}$, the association between leptin and HRV was not significant. However, among officers with $\mathrm{BMI} \geq 25 \mathrm{~kg} / \mathrm{m}^{2}$, the association between leptin and HRV was inversely related, after adjustment for age, gender, and race/ethnicity; p-values for trend (HF HRV, p $=0.019$ and LF HRV, $\mathrm{p}<0.0001)$. Similarly, among officers with percent body fat $\geq 25.5 \%$, leptin and LF HRV showed significant, inverse associations (adjusted $\mathrm{p}$ for trend $=0.001$ ).

Conclusions Our results show that leptin levels were inversely and significantly associated with HRV among all officers, and particularly among officers with higher levels of adiposity. These results suggest that increased leptin levels may be associated with CVD-related health problems.

\section{QUALITATIVE FINDINGS FROM A SAFETY COMMUNICATION AND RECOGNITION PROGRAM ON SAFETY AWARENESS AND TEAMBUILDING IN CONSTRUCTION}

${ }^{1}$ Emily H Sparer, ${ }^{2}$ Mia R Goldwasser, ${ }^{2}$ Kincaid A Lowe, ${ }^{1}$ Robert $F$ Herrick, 1,2Jack T Dennerlein. 'Harvard School of Public Health, Boston, MA, USA; ${ }^{2}$ Northeastern University, Boston, MA, USA

\subsection{6/oemed-2014-102362.205}

Objectives To qualitatively explore the impact of a safety communication and recognition program ("B-SAFE") on safety attitudes and beliefs among construction workers.

Method B-SAFE consisted of weekly, detailed feedback to foremen and workers on safe and unsafe work practices. B-SAFE ran for approximately 5 months on three commercial construction sites in Eastern Massachusetts. Sites were paired with a similar worksite (and same owner or general contractor), and data collection methods were identical at each site. Focus groups and key informant interviews were conducted to qualitatively assess the program's impact on workers' perception of site safety. Transcripts of focus groups and key informant interviews were coded and analysed for thematic content using Atlas.ti (V7).

Results At B-SAFE intervention sites, workers noted increased levels of safety awareness, communication, and teamwork, when compared to experiences on-site before the program, and to past worksites. Workers attributed an increase in morale to B-SAFE, noting that increasing safety performance feedback helped to improve safety conditions. One worker stated, "[B-SAFE] increased the level of awareness around safety conditions on-site (...) Instead of cutting corners, we'd do it right." Workers at sites without B-SAFE noted that the safety level was comparable to past worksites.

Conclusions The B-SAFE program led to many positive changes on-site, including an increase in safety awareness, teambuilding, and collaborative competition. Future quantitative data analysis to evaluate program effectiveness including worker surveys, safety inspections, and injury reports will augment these qualitative results.

\section{EPIDEMIOLOGICAL STUDY OF LUNG INFLAMMATION AND OXIDATIVE DAMAGE IN INDIUM TIN OXIDE WORKERS}

${ }^{1}$ Wei-Te Wu, ${ }^{1} Y u-T e h$ Chung, ${ }^{1}$ Hui-Yi Liao, ${ }^{2}$ Ching-Huang Lai, ${ }^{3}$ Chang-Yuh Chen, ${ }^{3}$ Lien-Hsiung Lee, ${ }^{3}$ Chiou-Jong Chen, ${ }^{1,2}$ Saou-Hsing Liou. ${ }^{1}$ Division of Environmental Health and Occupational Medicine, National Health Research Institutes, Miaoli County, Taiwan; ${ }^{2}$ Department of Public Health, National Defense Medical Center, Taipei, Taiwan; 3 Institute of Occupational Safety and Health, Council of Labor Affairs, Taipei, Taiwan

\subsection{6/oemed-2014-102362.206}

Objectives Indium Tin Oxide (ITO) is widely used in many kinds of touch panels nowadays. Workers could expose to ITO particles from sintering granules, splashing, pulverisation, cutting, and grinding processes. This study aimed to assess the relationship between ITO exposure and lung inflammation and oxidative damage in ITO workers.

Method We recruited 148 exposed workers and 38 control workers from ITO powder process, recycling and ITO target manufacturing plants in Taiwan. Indium in serum (S-In) and urine (U-In) was determined as biomarkers of exposure. Exposed group was further divided as high (S-In $>3 \mu \mathrm{g} / \mathrm{L}$ and low exposed groups (S-In $£ 3 \mu \mathrm{g} / \mathrm{L}$ ). Urinary and plasma 8-hydroxy-2deoxyguanosine (8-OHdG), serum Clara cell protein (CC16), and fractional exhaled nitric oxide (FENO) were measured as biomarkers of oxidative damage and pulmonary inflammation, respectively.

Results The geometric mean air concentrations of indium were $0.0041 \pm 2.49 \mathrm{mg} / \mathrm{m}^{3}$ by area sampling and $0.017 \pm 5.20 \mathrm{mg} /$ $\mathrm{m}^{3}$ by personal sampling. The mean S-In level and U-In level in high exposed group were $8.01 \mathrm{ppb}$ and $3.45 \mathrm{ppb}$, respectively. The mean levels of S-In and U-In in high exposed group were significantly higher than those of low exposed group. The mean levels of serum CC16 and urinary 8-OHdG in high exposed group were also significantly higher than those of low exposed groups. After adjusting potential confounders, dose-response gradients were found between S-In and CC16 ( $p=0.020)$ and between S-In and urinary 8-OHdG $(\mathrm{p}=0.027)$, respectively. Conclusions We concluded that indium particles exposure may induce lung inflammation and DNA oxidative damage.

\section{ROTATING NIGHT SHIFT WORK IN NURSES AND MIDWIVES AND LIFESTYLE}

Beata Peplonska, Weronika Burdelak, Agnieszka Bukowska, Wojciech Sobala. Nofer Institute of Occupational Medicine, Lodz, Poland

\subsection{6/oemed-2014-102362.207}

Objectives To investigate the association between rotating night shift work and selected modifiable lifestyle factors among nurses and midwives.

Method The cross-sectional study included 725 nurses and midwives aged 40-60 (354 rotating night shift and 371 daytime workers). Occupational history and data about potential confounders were collected through in-person interview. Weight and height were measured and BMI was calculated. Associations between night shift work characteristics such as current rotating 